\title{
TECNOLOGÍA DE ASISTENCIA: EXOESQUELETOS ROBÓTICOS EN REHABILITACIÓN
}

\section{ASSISTIVE TECHNOLOGY: ROBOTIC EXOSKELETONS IN REHABILITATION}

\author{
Jose Iván Alfonso-Mantilla ${ }^{1}$ \\ Jaime Martínez-Santa ${ }^{2}$
}

Fecha de Recepción: 12 de mayo de 2016

Fecha de Aceptación: 18 de diciembre de 2016

Citar como:

Alfonso-Mantilla J, Martínez-Santa J. (2016). Tecnología de asistencia: exoesqueletos robóticos en rehabilitación. Revista Movimiento Científico 10(2): 83-90.

Alfonso-Mantilla J, Martínez-Santa J. (2016). Tecnología de asistencia: exoesqueletos robóticos en rehabilitación. Revista Movimiento Científico [en línea] 2016, [fecha de consulta: dd//mm/aaaa]; 10(2): 83-90. Disponible desde: http://revistas.iberoamericana.edu.co/ index.php/Rmcientifico/issue/archive.

\section{RESUMEN}

Introducción: la robótica es la ciencia que combina la mecánica y electrónica; tiene como fin la creación de sistemas robóticos. Adicionalmente, esta área de conocimiento se especializa en la creación de tecnologías de asistencia para pacientes con enfermedades neurológicas como lesiones espinales, accidentes cerebrovasculares y enfermedades degenerativas de la médula espinal, estos sistemas son conocidos como exoesqueletos robóticos los cuales son sistemas mecatrónicos usados por una persona de tal manera que la interfaz física permite una transferencia directa de energía mecánica e intercambio de información. Objetivo: realizar una revisión de literatura acerca de los tipos de exoesqueletos robóticos y los beneficios de la intervención realizados con los mismos en pacientes con lesiones neurológicas. Materiales y Método: se realizó una revisión de la literatura en las siguientes bases de datos como: Ebsco, Pedro, Hinari, Elsevier, Science Direct, Springer Medline. Tuvo como criterios de inclusión estudios entre el 2000 y el 2016, con los siguientes términos DeCS: Exoskeleton device, rehabilitation, robotics, gait el idioma de los artículos consultados podía ser en español, inglés o portugués. Resultados: existen diferentes tipos de exoesqueletos robóticos tanto de miembros inferiores y superiores, estos utilizan una interfaz entre el sujeto para obtener una interacción mecánica, en enfermedades neurológicas como lesiones espinales y accidentes cerebrovasculares se ha reportado el aumento de variables cinéticas y cinemáticas en el patrón de marcha así como el aumento funcionalidad

Fisioterapeuta. Universidad del Rosario. Correo electrónico: josealfonso25@hotmail.com

Fisioterapeuta. Universidad Nacional de Colombia. Especialista en Epidemiología. Universidad del Rosario. Especialista en Estadística.

Universidad Nacional. Correo electrónico: jmartinezsanta@yahoo.com 
en mano. Conclusiones: los exoesqueletos robóticos deben ser el futuro de intervenciones en fisioterapia debido a su alto nivel de confiabilidad.

Palabras clave: Dispositivo exoesqueleto, rehabilitación, robótica, marcha.

\section{ABSTRACT}

Background: Robotics is the science that combines mechanics and electronics. Its purpose is to create robotic systems. Additionally, this area of expertise specializes in the creation of assistive technologies for patients with neurological diseases such as spinal injuries, cerebrovascular accidents and degenerative diseases of the spinal cord, these systems are known as robotic exoskeletons which are mechatronic systems used by a Person in such a way that the physical interface allows a direct transfer of mechanical energy and the exchange of information. Objective: literature review about the types of robotic exoskeletons and the benefits of the intervention performed with them in patients with neurological injuries. Materials and Methods: A review of the literature was carried out in the following databases: "Ebsco" "Pedro" "Hinari" "Elsevier" "Science Direct" "Springer" "Medline". It had as inclusion criteria studies between 2000 and 2016, with the following terms DeCS: Exoskeleton device, rehabilitation, robotics, gait, the language of the articles consulted could be in Spanish, English or Portuguese. Results: There are different types of robotic exoskeletons of both lower and upper limbs, these use an interface between the subject to obtain a mechanical interaction, in neurological diseases such as spinal lesions and cerebrovascular accidents has been reported the increase of kinetic and kinematic variables in the pattern As well as increasing functionality in hand. Conclusions: Robotic exoskeletons should be the future of physiotherapy interventions because of their high level of reliability.

Keywords: Exoskeleton device, rehabilitation, robotics, gait.

\section{INTRODUCGIÓN}

La robótica es la ciencia que combina la mecánica, electrónica, informática e inteligencia artificial, esta tiene como fin la creación de sistemas robóticos. Adicionalmente, esta área de conocimiento se especializa en la creación de tecnologías de asistencia para pacientes con enfermedades neurológicas como lesiones espinales, accidentes cerebrovasculares y enfermedades degenerativas de la médula espinal; estos sistemas son conocidos como exoesqueletos robóticos los cuales son sistemas mecatrónicos usados por una persona de tal manera que la interfaz física permite una transferencia directa de energía mecánica y el intercambio de información, lo cual lo convierten en dispositivos de asistencia que incrementan la funcionalidad de las extremidades superiores e inferiores (Chen, Chan, Guo, \& Yu, 2013; Fisahn et al., 2016; Lajeunesse, Vincent, Routhier, Careau, \& Michaud, 2016).
Los exoesqueletos son una herramienta de innovación en el proceso de rehabilitación, se ha reportado el uso de exoesqueletos robóticos como tecnología de asistencia que potencia las cualidades físicas como: fuerza, resistencia, potencia y flexibilidad, además de proporcionar retroalimentación cuantitativa y aumentar los resultados funcionales en la recuperación de pacientes con lesiones neurológicas (Chen et al., 2013; Ferrigno et al., 2011).

Los exoesqueletos son conocidos como exomarco o exotraje y están basados en diseños mecánicos construidos con motores hidráulicos que ayudan a su portador a realizar movimientos con más potencia y fuerza (Bortole et al., 2015; Ferrigno et al., 2011; Olaya, 2009). Además, su funcionamiento depende de una serie de sensores biométricos que detectan señales nerviosas que el cerebro envía a los músculos para desarrollar determinada acción. El exoesqueleto codifica y realiza las acciones enviadas por estos 
impulsos nerviosos, lo cual promueve la plasticidad cerebral en pacientes con daño neurológico y permite recuperar la locomoción (Bortole et al., 2015; Ferrigno et al., 2011; Olaya, 2009)

En la actualidad, se pueden encontrar diferentes tipos de exoesqueletos usados en rehabilitación tales como el Stride de Honda, Argo medical, Hal de Tsukuba, Anklebot de MIT y Re walk technologies usados para procesos de rehabilitación de la locomoción en paciente con lesiones neurológicas (Bortole et al., 2015; Ferrigno et al., 2011).

La expansión en este campo de investigación, aumenta a un ritmo exponencial las posibilidades de intervención en fisioterapia basadas en sistemas robóticos.

Los estudios han determinado que entre los usos de los exoesqueletos se encuentran: aumentar el rendimiento humano, la movilidad de individuos con patologías neurológicas y brindar tecnología de asistencia para personas en situación de discapacidad (Louie \& Eng, 2016; Miller, Zimmermann, \& Herbert, 2016). El futuro de esta tecnología está enmarcado en la investigación de distintas áreas del saber cómo informática, robótica y rehabilitación (Ferrigno et al., 2011; Ferris, 2010). El objetivo de este artículo fue realizar una revisión de literatura acerca de los tipos de exoesqueletos robóticos y los beneficios de la intervención realizados con los mismos en pacientes con lesiones neurológicas.

\section{MATERIALES Y MÉTODOS}

Se realizó una revisión de la literatura en las siguientes bases de datos: Ebsco, Pedro, Hinari, Elsevier, Science Direct, Springer, Medline.

Se consideraron los siguientes criterios de inclusión y exclusión.

Inclusión: fueron incluidos artículos que su año de publicación fuera del 2000 al 2016, literatura con los siguientes términos DeCS: Exoskeleton device, rehabilitation, robotics, gait. Idioma de los artículos consultados podía ser español, inglés o portugués. Se incluyeron en la búsqueda ensayos clínicos aleatorizados (ECA), Metaanálisis, casos y controles, estudios de cohorte, revisiones sistemáticas y revisiones de literatura.

Exclusión: estudios que su año de publicación fuera inferior al año 2000, que no contemplaran los términos DeCS establecidos, estudios que no estuvieran disponibles o completos, estudios que se relacionaron con tecnología humanoide.

\section{RESULTADOS}

En la figura 1 se ilustra el proceso de extracción de la evidencia en las bases de datos

Figura 1. Flujograma de extracción de la evidencia

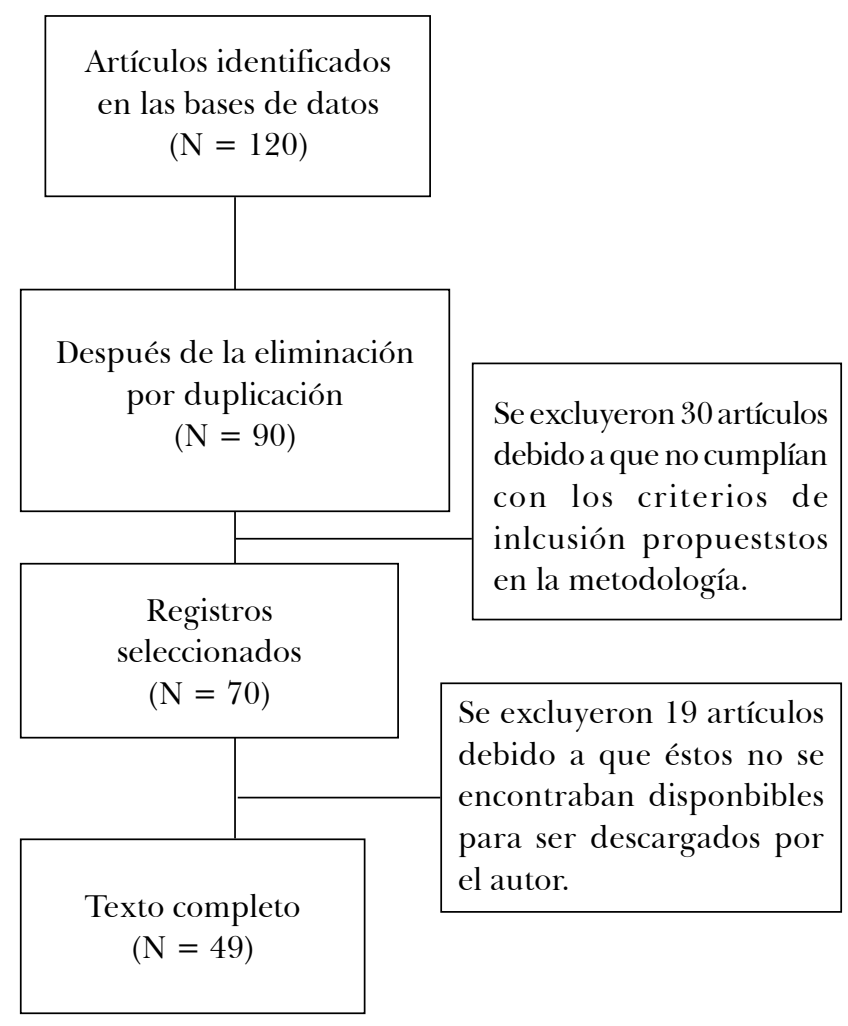

Fuente de elaboración propia (2016)

Dentro de la revisión efectuada se pudieron identificar diferentes tipos de exoesqueletos de miembros inferiores y superiores utilizados en rehabilitación así como los beneficios de trabajar con los mismos. 


\section{Tipos de exoesqueletos}

El WABIAN-RIV tiene 43 grados de libertad; 6 en miembros inferiores, 7 en miembros superiores, 3 en manos, 2 en ojos, 4 en cuello y 3 en cintura; su altura es de 1,89 metros y su peso es de $127 \mathrm{Kg}$. Contiene un sistema de visión y reconocimiento (Lim \& Takanishi, 2007).

El WL-16 consiste en una pelvis y dos piernas que tienen 6 grados de movimiento los cuales actúan en un plano lineal, con una silla de aluminio que reduce las cargas durante la fase de apoyo de la marcha. Contiene un soporte de reducción de cargas compuesto de resortes de compresión hidráulica. Estos modelos permiten procesos de rehabilitación de marcha, locomoción y balance. Adicionalmente, poseen sistemas para el trabajo en miembros superiores(Lim \& Takanishi, 2007).

El exoesqueleto WOTAS (Órtesis portátil para la evaluación de temblores y represión) tiene altos índices de confiabilidad y validez en la prevención de temblores en pacientes neurológicos a través de la aplicación de fuerzas internas (Agrawal et al., 2007; Kao, Lewis, \& Ferris, 2010a, 2010b, 2010c; Lewis \& Ferris, 2011; Tang et al., 2014).

El exoesqueleto MAHI Exo-II es especializado en la rehabilitación de extremidades superiores de pacientes con alteraciones neurológicas, el entrenamiento de larga duración y alta intensidad permite la recuperación motora de estos pacientes (Bortole et al., 2015; French, Rose, \& O'Malley, 2014; Pehlivan, Rose, \& O'Malley, 2013; Reinkensmeyer, Akoner, Ferris, \& Gordon, 2009).

El exoesqueleto $\mathrm{H} 2$ cuenta con seis articulaciones y está diseñado para incrementar funcionalidad de pacientes con lesiones neurológicas; es utilizado en pacientes con accidente cerebrovascular a quienes se les realizó un tratamiento de 12 sesiones durante 4 semanas logrando un aumento de la movilidad y realización de tareas de la vida diaria (Bortole et al., 2015; French et al., 2014; Pehlivan et al., 2013; Reinkensmeyer et al., 2009).

\section{Creación de los exoesqueletos}

Para la creación de exoesqueletos robóticos se usan patrones electrónicos e informáticos, los cuales ofrecen cualidades de grados de movimiento y permiten la adaptación y generación de movimiento en estos sistemas.

Además, los exoesqueletos utilizados en rehabilitación poseen mecanismos elásticos poliarticulares los cuales se componen de doce poleas para los miembros inferiores capaces de generar fuerza muscular y ayudar a la locomoción del paciente; estos exoesqueletos se construyen con accionamiento neumático, lo cual reduce la carga biomecánica y muscular sobre el paciente (Renjewski \& Seyfarth, 2012; Sawicki \& Ferris, 2008, 2009; Van den Bogert, 2003).

Los sistemas de control de los exoesqueletos deben realizarse a través de algoritmos que identifiquen las fases de la marcha y permitan una adaptación a diferentes condiciones para la locomoción, estos deben basarse en controladores que se adapten a modelos biomecánicas de locomoción humana debido a que el uso de los mismos permite el aumento de fuerza en grupos musculares específicos y permite realizar evaluaciones de la marcha a nivel cinético y cinemático (Hornby et al., 2012; Li et al., 2014; Sylos-Labini et al., 2014).

\section{Usos y beneficios de los exoesqueletos en rehabilitación}

En pacientes neurológicos con enfermedades como accidentes cerebrovasculares y lesiones medulares el uso de los exoesqueletos ha demostrado tener altos índices de efectividad debido a que los sistemas hidráulicos permiten generar fuerza a través de torques y sistemas biomecánicos involucrados en la marcha de estos pacientes (Jimenez-Fabian \& Verlinden, 2012; Kao et al., 2010a, 2010b; Koller, Jacobs, Ferris, \& Remy, 2015; Sawicki \& Ferris, 2009).

Se ha referenciado que el uso de exoesqueletos debe ser aproximadamente de 30 minutos para ayudar al paciente a realizar procesos de recuperación más 
efectivos con el fin de alcanzar rangos de movimiento funcionales; esto se da a través de la contracción muscular debido al reclutamiento de las fibras musculares y la activación del órgano tendinoso de Golgi (Fleischer, Wege, Kondak, \& Hommel, 2006; Krebs et al., 2007; Rocon et al., 2007). Es por eso que la utilización de exoesqueletos es un conjunto de conocimientos a nivel biológico, biomecánico, electrónico que muestra resultados efectivos en la atención de pacientes con alteración neurológica (Hornby et al., 2012; Li et al., 2014; Sylos-Labini et al., 2014).

Las enfermedades neurológicas afectan la movilidad y el desarrollo de actividades de la vida diaria de las personas que la padecen. El uso de exoesqueletos robóticos en pacientes con lesiones espinales crónicas demuestra tener resultados significativos en la velocidad del patrón de marcha, aumento del consumo de oxígeno y mayor tolerancia de variables hemodinámicas ante el esfuerzo físico (Evans, Hartigan, Kandilakis, Pharo, \& Clesson, 2015).

Adicionalmente, se reporta el aumento en variables cinéticas y cinemáticas de la marcha tales como: velocidad, distancia, longitud de zancada, ancho de paso, cadencia (Kozlowski, Bryce, \& Dijkers, 2015; Yang, Asselin, Knezevic, Kornfeld, \& Spungen, 2015). De hecho, el uso de exoesqueletos aumenta la habilidad para mantener el balance mientras la persona está en posición bípeda, aumenta la propiocepción además de mejorar los traslados y la descarga de pesos en extremidades inferiores, aumenta en rango de movimiento en cadera, rodilla y cuello de pie (Aach et al., 2014; Asselin, Avedissian, Knezevic, Kornfeld, \& Spungen, 2016; Esquenazi, Talaty, Packel, \& Saulino, 2012; Sczesny-Kaiser et al., 2015).

En patologías como accidentes cerebrovasculares el uso de exoesqueletos ha reportado aumento en parámetros de la marcha, aumento de fuerza del lado afectado de la lesión cerebral (Alavi, Herrnstadt, Randhawa, Boyd, \& Menon, 2015; Buesing et al, 2015). En este tipo de lesiones son comunes el uso de exoesqueletos de miembros superiores los cuales se basan en rehabilitación de codo y mano a través de sistemas de poleas que favorecen agarres y alcances funcionales (Alavi et al., 2015; Zhang, Fu, Zhang, \& Wang, 2015).

Se reporta que el uso de exoesqueletos puede ser de 8 semanas de tratamiento, 20 minutos por sesión donde se combinen ejercicios de marcha. Los resultados de la intervención con exoesqueletos pueden ser medidos a través de escalas como: 10 metros, time up and go, test de 6 minutos, funcional reach test y escala de BERG (Yoshimoto, Shimizu, \& Hiroi, 2016; Yoshimoto et al., 2015).

\section{DISCUSIÓN}

A lo largo del texto se evidencia que el uso de exoesqueletos en rehabilitación tiene efectos significativos en patologías neurológicas como eventos cerebrovasculares, lesiones espinales y algunos procesos de alteraciones osteomusculares donde a través de estos sistemas de promueve la recuperación de la funcionalidad de estos pacientes(Cooper et al., 2008). De igual manera estudios revelan que las personas mayores de 65 años se duplicarán entre el año 19972025, y la discapacidad crecerá a un ritmo similar por eso se necesitará que este tipo de tecnologías robóticas sea investigada y aplicada para poder desarrollar procesos de rehabilitación (Cooper et al., 2008).

Muchos estudios han reportado que la utilización de equipos de asistencia híbridos tienen beneficios en la activación de los sistemas sensoriales y motores de individuos afectados con lesiones neurológicas al favorecer la plasticidad neuronal para incrementar su recuperación y funcionalidad (Popovic \& Popovic, 2006); es por esto, que el futuro está enmarcado en el uso de exoesqueletos robóticos para los procesos de rehabilitación de pacientes con lesiones neurológicas, pues demuestran ser efectivos y tener altos índices de confiabilidad, el papel del fisioterapeuta es adecuar su conocimiento al uso de estos nuevos sistemas de intervención con el fin de utilizar la tecnología como su principal herramienta de acción (Francis \& Winfield, 2006; Lo \& Xie, 2012). 
Investigaciones futuras en fisioterapia e ingeniería biomédica deben estar encaminadas hacia la creación de exoesqueletos robóticos autosustentables y de fácil acceso a población de bajos recursos (Gillesen, Barakova, Huskens, \& Feijs, 2011; Masiero et al., 2009; Yakub, Md Khudzari, \& Mori, 2014).

\section{CONGLUSIONES}

Los exoesqueletos robóticos son una herramienta de intervención en fisioterapia que demuestran tener resultados significativos en pacientes con enfermedades neurológicas como lesiones espinales y accidentes cerebrovasculares, con ayuda de los exoesqueletos mejora la locomoción, balance y propiocepción.

Los exoesqueletos robóticos deben ser el futuro de intervenciones en fisioterapia debido a su alto nivel de confiabilidad.

\section{LIMITACIONES Y CAMINOS FUTUROS}

En Colombia este tipo de tecnología debería estar al alcance de toda la población con lesiones neurológicas para favorecer su recuperación funcional y garantizar su retorno a las actividades de la vida diaria. En contraste, las entidades en salud deben poner al alcance de la población todas las herramientas posibles para su recuperación después de una lesión.

\section{REFERENCIAS}

Aach, M., Cruciger, O., Sczesny-Kaiser, M., Hoffken, O., Meindl, R., Tegenthoff, M., et al. (2014). Voluntary driven exoskeleton as a new tool for rehabilitation in chronic spinal cord injury: a pilot study. Spine J, 14(12), 2847-2853.

Agrawal, S. K., Banala, S. K., Fattah, A., Sangwan, V., Krishnamoorthy, V., Scholz, J. P., \& Hsu, W. L. (2007). Assessment of motion of a swing leg and gait rehabilitation with a gravity balancing exoskeleton. IEEE Trans Neural Syst Rehabil Eng, 15(3), 410-420.

Alavi, N., Herrnstadt, G., Randhawa, B. K., Boyd, L. A., \& Menon, C. (2015). Bimanual elbow exoskeleton: Force based protocol and rehabilitation quantification. Conf Proc IEEE Eng Med Biol Soc, 2015, 4643-4646.
Asselin, P. K., Avedissian, M., Knezevic, S., Kornfeld, S., \& Spungen, A. M. (2016). Training Persons with Spinal Cord Injury to Ambulate Using a Powered Exoskeleton. $J$ Vis $\operatorname{Exp}(112)$.

Bortole, M., Venkatakrishnan, A., Zhu, F., Moreno, J. C., Francisco, G. E., Pons, J. L., \& Contreras-Vidal, J. L. (2015). The H2 robotic exoskeleton for gait rehabilitation after stroke: early findings from a clinical study.J Neuroeng Rehabil, 12, 54.

Buesing, C., Fisch, G., O'Donnell, M., Shahidi, I., Thomas, L., Mummidisetty, C. K., et al. (2015). Effects of a wearable exoskeleton stride management assist system $(\mathrm{SMA}(\mathrm{R}))$ on spatiotemporal gait characteristics in individuals after stroke: a randomized controlled trial. J Neuroeng Rehabil, 12, 69.

Chen, G., Chan, C. K., Guo, Z., \& Yu, H. (2013). A review of lower extremity assistive robotic exoskeletons in rehabilitation therapy. Crit Rev Biomed Eng, 41(4-5), 343-363.

Cooper, R. A., Dicianno, B. E., Brewer, B., LoPresti, E., Ding, D., Simpson, R., et al. (2008). A perspective on intelligent devices and environments in medical rehabilitation. Med Eng Phys, 30(10), 1387-1398.

Esquenazi, A., Talaty, M., Packel, A., \& Saulino, M. (2012). The ReWalk powered exoskeleton to restore ambulatory function to individuals with thoracic-level motor-complete spinal cord injury. Am J Phys Med Rehabil, 91(11), 911-921.

Evans, N., Hartigan, C., Kandilakis, C., Pharo, E., \& Clesson, I. (2015). Acute Cardiorespiratory and Metabolic Responses During Exoskeleton-Assisted Walking Overground Among Persons with Chronic Spinal Cord Injury. Top Spinal Cord Inj Rehabil, 21(2), 122-132.

Ferrigno, G., Baroni, G., Casolo, F., De Momi, E., Gini, G., Matteucci, M., \& Pedrocchi, A. (2011). Medical robotics. IEEE Pulse, 2(3), 55-61.

Ferris, P. (2010). Robotic lower limb orthosis: goals obstacles and current research. Paper presented at the The 34 th Annual Meerting of the American Sociaty of Biomechanics, Symposia: Robotic Lower Limb Ortheses and Prostheses.

Fisahn, C., Aach, M., Jansen, O., Moisi, M., Mayadev, A., Pagarigan, K. T., et al. (2016). The Effectiveness and Safety of Exoskeletons as Assistive and Rehabilitation Devices in the Treatment of Neurologic Gait Disorders in Patients with Spinal Cord Injury: A Systematic Review. Global Spine J, 6(8), 822-841.

Fleischer, C., Wege, A., Kondak, K., \& Hommel, G. (2006). Application of EMG signals for controlling exoskeleton robots. Biomed Tech (Berl), 51(5-6), 314-319. 
Francis, P., \& Winfield, H. N. (2006). Medical robotics: the impact on perioperative nursing practice. Urol Nurs, 26(2), 99-104, 107-108.

French, J. A., Rose, C. G., \& O'Malley, M. K. (2014). System Characterization of MAHI EXO-II: A Robotic Exoskeleton for Upper Extremity Rehabilitation. Proc ASME Dyn Syst Control Conf, 2014.

Gillesen, J. C., Barakova, E. I., Huskens, B. E., \& Feijs, L. M. (2011). From training to robot behavior: towards custom scenarios for robotics in training programs for ASD. IEEE Int Conf Rehabil Robot, 2011, 5975381.

Hornby, T. G., Kinnaird, C. R., Holleran, C. L., Rafferty, M. R., Rodriguez, K. S., \& Cain, J. B. (2012). Kinematic, muscular, and metabolic responses during exoskeletal-, elliptical-, or therapist-assisted stepping in people with incomplete spinal cord injury. Phys Ther, 92(10), 1278-1291.

Jimenez-Fabian, R., \& Verlinden, O. (2012). Review of control algorithms for robotic ankle systems in lower-limb orthoses, prostheses, and exoskeletons. Med Eng Phys, 34(4), 397-408.

Kao, P. C., Lewis, C. L., \& Ferris, D. P. (2010a). Invariant ankle moment patterns when walking with and without a robotic ankle exoskeleton. J Biomech, 43(2), 203-209.

Kao, P. C., Lewis, C. L., \& Ferris, D. P. (2010b). Joint kinetic response during unexpectedly reduced plantar flexor torque provided by a robotic ankle exoskeleton during walking. J Biomech, 43(7), 1401-1407.

Kao, P. C., Lewis, C. L., \& Ferris, D. P. (2010c). Short-term locomotor adaptation to a robotic ankle exoskeleton does not alter soleus Hoffmann reflex amplitude.J Neuroeng Rehabil, 7, 33.

Koller, J. R., Jacobs, D. A., Ferris, D. P., \& Remy, C. D. (2015). Learning to walk with an adaptive gain proportional myoelectric controller for a robotic ankle exoskeleton. $J$ Neuroeng Rehabil, 12, 97.

Kozlowski, A. J., Bryce, T. N., \& Dijkers, M. P. (2015). Time and Effort Required by Persons with Spinal Cord Injury to Learn to Use a Powered Exoskeleton for Assisted Walking. Top Spinal Cord Inj Rehabil, 21(2), 110-121.

Krebs, H. I., Volpe, B. T., Williams, D., Celestino, J., Charles, S. K., Lynch, D., \& Hogan, N. (2007). Robot-aided neurorehabilitation: a robot for wrist rehabilitation. IEEE Trans Neural Syst Rehabil Eng, 15(3), 327-335.

Lajeunesse, V., Vincent, C., Routhier, F., Careau, E., \& Michaud, F. (2016). Exoskeletons' design and usefulness evidence according to a systematic review of lower limb exoskeletons used for functional mobility by people with spinal cord injury. Disabil Rehabil Assist Technol, 11(7), 535-547.
Lewis, C. L., \& Ferris, D. P. (2011). Invariant hip moment pattern while walking with a robotic hip exoskeleton. J Biomech, 44(5), 789-793.

Li, Z., Wang, B., Sun, F., Yang, C., Xie, Q., \& Zhang, W. (2014). sEMG-based joint force control for an upper-limb power-assist exoskeleton robot. IEEE J Biomed Health Inform, 18(3), 1043-1050.

Lim, H. O., \& Takanishi, A. (2007). Biped walking robots created at Waseda University: WL and WABIAN family. Philos Trans A Math Phys Eng Sci, 365(1850), 49-64.

Lo, H. S., \& Xie, S. Q. (2012). Exoskeleton robots for zupper-limb rehabilitation: state of the art and future prospects. Med Eng Phys, 34(3), 261-268.

Louie, D. R., \& Eng, J. J. (2016). Powered robotic exoskeletons in post-stroke rehabilitation of gait: a scoping review. J Neuroeng Rehabil, 13(1), 53.

Masiero, S., Carraro, E., Ferraro, C., Gallina, P., Rossi, A., \& Rosati, G. (2009). Upper limb rehabilitation robotics after stroke: a perspective from the University of Padua, Italy. J Rehabil Med, 41(12), 981-985.

Miller, L. E., Zimmermann, A. K., \& Herbert, W. G. (2016). Clinical effectiveness and safety of powered exoskeleton-assisted walking in patients with spinal cord injury: systematic review with meta-analysis. Med Devices (Auckl), 9, 455-466.

Olaya, A. F. R. (2009). Sistema robótico multimodal para análisis y estudios en biomecánica, movimiento humano y control neuromotor. Universidad Carlos III de Madrid.

Pehlivan, A. U., Rose, C., \& O'Malley, M. K. (2013). System characterization of RiceWrist-S: a forearm-wrist exoskeleton for upper extremity rehabilitation. IEEE Int Conf Rehabil Robot, 2013, 6650462.

Popovic, D. B., \& Popovic, M. B. (2006). Hybrid assistive systems for rehabilitation: lessons learned from functional electrical therapy in hemiplegics. Conf Proc IEEE Eng Med Biol Soc, 1, 2146-2149.

Reinkensmeyer, D. J., Akoner, O., Ferris, D. P., \& Gordon, K. E. (2009). Slacking by the human motor system: computational models and implications for robotic orthoses. Conf Proc IEEE Eng Med Biol Soc, 2009, 2129-2132.

Renjewski, D., \& Seyfarth, A. (2012). Robots in human biomechanics-a study on ankle push-off in walking. Bioinspir Biomim, 7(3), 036005.

Rocon, E., Belda-Lois, J. M., Ruiz, A. F., Manto, M., Moreno, J. C., \& Pons, J. L. (2007). Design and validation of a rehabilitation robotic exoskeleton for tremor assessment and suppression. IEEE Trans Neural Syst Rehabil Eng, 15(3), 367-378. 
Sawicki, G. S., \& Ferris, D. P. (2008). Mechanics and energetics of level walking with powered ankle exoskeletons. J Exp Biol, 211(Pt 9), 1402-1413.

Sawicki, G. S., \& Ferris, D. P. (2009). Powered ankle exoskeletons reveal the metabolic cost of plantar flexor mechanical work during walking with longer steps at constant step frequency. J Exp Biol, 212(Pt 1), 21-31.

Sczesny-Kaiser, M., Hoffken, O., Aach, M., Cruciger, O., Grasmucke, D., Meindl, R.,... Tegenthoff, $M$. (2015). HAL(R) exoskeleton training improves walking parameters and normalizes cortical excitability in primary somatosensory cortex in spinal cord injury patients. $J$ Neuroeng Rehabil, 12, 68.

Sylos-Labini, F., La Scaleia, V., d'Avella, A., Pisotta, I., Tamburella, F., Scivoletto, G., ... Ivanenko, Y. P. (2014). EMG patterns during assisted walking in the exoskeleton. Front Hum Neurosci, 8, 423.

Tang, Z., Zhang, K., Sun, S., Gao, Z., Zhang, L., \& Yang, Z. (2014). An upper-limb power-assist exoskeleton using proportional myoelectric control. Sensors (Basel), 14(4), 6677-6694.

van den Bogert, A. J. (2003). Exotendons for assistance of human locomotion. Biomed Eng Online, 2, 17.
Yakub, F., Md Khudzari, A. Z., \& Mori, Y. (2014). Recent trends for practical rehabilitation robotics, current challenges and the future. Int J Rehabil Res, 37(1), 9-21.

Yang, A., Asselin, P., Knezevic, S., Kornfeld, S., \& Spungen, A. M. (2015). Assessment of In-Hospital Walking Velocity and Level of Assistance in a Powered Exoskeleton in Persons with Spinal Cord Injury. Top Spinal Cord Inj Rehabil, 21(2), 100-109.

Yoshimoto, T., Shimizu, I., \& Hiroi, Y. (2016). Sustained effects of once-a-week gait training with hybrid assistive limb for rehabilitation in chronic stroke: case study. $J$ Phys Ther Sci, 28(9), 2684-2687.

Yoshimoto, T., Shimizu, I., Hiroi, Y., Kawaki, M., Sato, D., \& Nagasawa, M. (2015). Feasibility and efficacy of high-speed gait training with a voluntary driven exoskeleton robot for gait and balance dysfunction in patients with chronic stroke: nonrandomized pilot study with concurrent control. Int J Rehabil Res, 38(4), 338-343.

Zhang, F., Fu, Y., Zhang, Q., \& Wang, S. (2015). Experiments and kinematics analysis of a hand rehabilitation exoskeleton with circuitous joints. Biomed Mater Eng, 26 Suppl 1, S665-672. 